\title{
Coping with adequate and inadequate self-assessment of older people's risk of falling: a mixed-methods study in Germany.
}

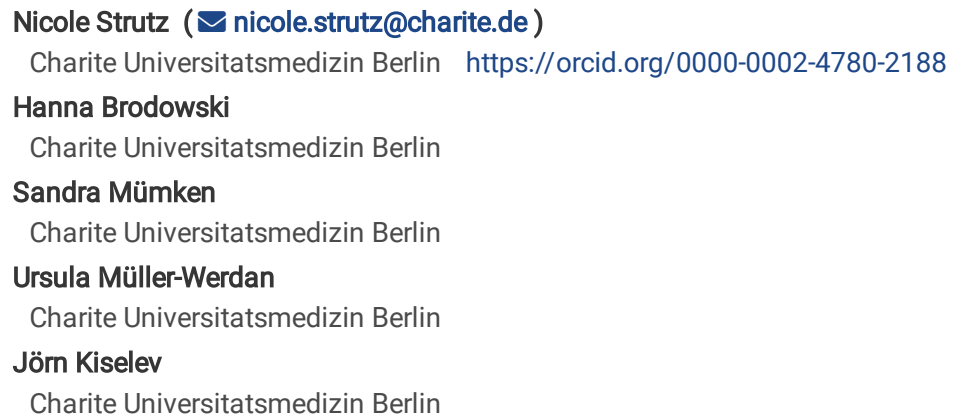

Research article

Keywords: Self-assessment, risk of falling, coping strategies, older people

Posted Date: December 10th, 2019

DOI: https://doi.org/10.21203/rs.2.18153/v1

License: (c) (7) This work is licensed under a Creative Commons Attribution 4.0 International License. Read Full License 


\section{Abstract}

Background: Older people are exposed to an increased risk of falling due to a multitude of physiological and functional changes and fear of falling. The risk of falling is assessed and managed differently. It should be found out whether the coping strategies of older people differ with regard to their activities in daily life depending on the adequacy of their self-assessed fall risk.

Methods: Adequacy of the perceived fall risk was evaluated with the de Morton Mobility Index and the Activities-specific Balance Confidence Scale among 100 older people. Additionally, semi-structured interviews were conducted in 16 participants with an identified fall risk. Coding techniques, based on structuring qualitative content analysis and the half-split method were applied.

Results: Six out of 16 interviewees assessed their fall risk adequately. Interviews with the seniors resulted in topics such as coping strategies and awareness and several sub-topics in each category. Participants who adequately assessed their own fall risk reported an "active/positive" coping behavior and awareness of themselves. In contrast, those who assessed their own risk of falling inadequately covered all identified categories with no identifiable pattern.

Conclusion: An adequate self-assessment of fall risks is accompanied by positive coping strategies to maintain an active lifestyle. In contrast, a lack of an adequate self-awareness seems to lead to arbitrary coping strategies. Assisting older people in their self-awareness regarding their own fall risk should be emphasized in order to adopt positive coping strategies. Future studies are necessary to better understand the underlying mechanisms that lead to an adequate or inadequate self-assessment in older people fall risk.

\section{Background}

Older people are exposed to increased fall risk (FR) because of a variety of physiological and functional changes, such as fading of muscle power, reduction in posture control and visual impairment, due to senescence (1). In Germany, falls are the most frequent cause of accidents; additionally, FR increases with increasing age (2). Of those aged 65 to 79 years, almost one third experience a fall at least once a year; of those aged 80 and above, that number rises to $50 \%$ (3). About $10-20 \%$ of all falls result in injuries, hospitalization or death (4). In the US, falls are among the 10 leading causes of death for people over the age of $65(4)$.

Based on the significance of falls, Filiatrault and Desroisiers (2009) addressed the topic of the fear of falling (FOF) and associated coping strategies (5). However, the adequacy of FOF was not considered in this work. Since then, several publications evaluated the adequacy of subjectively perceived FR in older people and the concordance of subjective perception with objective mobility measurements. Based on these studies, around $22-42 \%$ of older people are inadequately assessing their own FR (6-8). However, studies on the underlying reasons for the observed inadequacy or consequences due to this inadequacy are lacking. In 2011, Høst et al. examined the relationship between the adequate assessment of the risk of falling and accompanied coping strategies of older people (9). They identified an active coping strategy, which is characterized by retaining the unchanged behavior that existed right before the fall and a passive coping strategy, which serves as a fall avoidance technique (9). Høst et al. described passive coping strategies of those who have already fallen as follows: "[They] stopped doing certain activities to avoid falling and they did not choose activities that made them scared and nervous and caused bodily pain."

The concept of coping was defined by Lazarus et al. (1993) who postulated that it a) has a specific external or internal requirement, which is evaluated as a burden / an excess of the person's resources and b) includes a cognitive and behavioral component. Lazarus described this in terms of "ongoing cognitive and behavioral efforts to manage specific external and/or internal demands that are appraised as taxing or exceeding the resources of the person" (10). Furthermore, in their understanding, the term coping is independent of attributes such as adaptive/non-adaptive, successful/unsuccessful, or stable/variable.

Filiatraut and Desrosiers additionally came to the conclusion that, in the context of FOF among older people, coping as "a strategy involving excessive activity restriction could negatively impact a senior's health and quality of life and is likely to be perceived as unsatisfactory, especially when the activity is meaningful to the person" (5) (An additional file shows this in more detail [see Additional file 1]).

The present study was based on the concept of coping-especially the two coping strategies defined by Høst et al.-and examines whether the results are generalizable to geriatric patients on a German geriatric ward. In addition, it explores whether the objectively measured FR correlates with the self-assessed risk of falling on the part of participants. Finally, we aimed at identifying whether older people who self-assessed their risk of falling, both adequately and not, differed regarding their proposed coping strategies. For this, a subcohort of a larger cohort study on the consequences of FR and mobility in older people after a hospital stay was interviewed half a year after discharge from hospital.

In the context of this analysis, we tried to answer the following research questions:

- "Do older people who adequately or inadequately assess their risk of falling demonstrate different coping strategies?"

- "Which coping strategies are used by people from the age of 65 onwards who self-assess their risk of falling adequately or inadequately?"

\section{Data And Methods}

The aim of this study is to evaluate whether older people who adequately or inadequately assess their risk of falling demonstrate different coping strategies. And which coping strategies are used by these older people. The present study is a subcohort within a cohort study that adopts a mixed-method approach involving a sequential design. A quantitative approach right before the test person's discharge from a geriatric clinic was combined with a qualitative one six months after their discharge (see Figure 1) 


\section{FIGURE 1}

The decision to follow a sequential procedure was made in order to factor in qualitative answers about the participants' self-assessed FR-adequate or inadequate-during the interviews.

For the quantitative part, 138 patients were recruited consecutively in a geriatric hospital. Inclusion criteria were defined as follows: an age 65 years onwards, an expected ability to walk and climb stairs with the full load during the hospital stay, sufficient vision to complete a questionnaire, and the participant's ability to consent. Exclusion criteria were: the presence of severe heart failure (NYHA IV) or peripheral arterial occlusive disease (PADD) of the lower extremities (grade 3+), amputation of one or both lower extremities, poor understanding of the German language, severe cognitive deficits or mental illness, and the lack of written consent.

During the hospital stay, 38 of the 138 participants dropped out of the study. The reasons for dropout were: withdrawal of consent, inadequate response, early hospital discharge, deterioration in the general condition (physical or mental). Data collection lasted from September 2016 to March 2018.

Quantitative data collection was undertaken during the hospital stay. All participants were included within the first five days after admittance to hospital. Directly after inclusion, sociodemographic data of the study participants, as well as data on falls in the recent past 12 months, perceived general self-efficacy and social activities in the last six months were collected. Within three days before discharge, data on the confidence to maintain balance in everyday situations were recorded using the Activities-specific Balance Confidence Scale (ABC Scale). Additionally, the de Morton Mobility Index (DEMMI), a mobility test for geriatric patients whose score is associated with falls, was performed (T1).

The ABC Scale captures the subjective confidence to maintain balance without staggering during 16 everyday defined activities (e.g., climbing stairs, using an escalator) (11). The validated German version of the $A B C$ Scale was used (12). While the original scale evaluates the individual items on a scale between 0 and 100 points, the present study uses a Likert-scale version with the following response categories: $0=$ not at all confident, $1=$ a little confident, $2=$ confident, 3 = very confident (13). The overall score is thus in line with this survey, i.e., between 0 and 48 points. A cutoff value of less than $67 \%$ identified subjects with an increased FR (14). The ABC Scale shows a high test reliability (15) as well as a good discriminant and convergent validity (11).

The DEMMI was developed as a measurement tool for the assessment of the general mobility of the elderly, who are located in an acute care or geriatric rehabilitation facility (16). The 15 items of the DEMMI check the ability of a person to change position and location, with or without assistance from the investigator. Likewise, a person's walking and balancing abilities are tested. The items are hierarchically ordered according to the level of difficulty (16). The addition of all 15 items' ratings gives a raw score with a range of $0-18$, which is then transformed into a score with a span of $0-100$. Zero indicates the lowest and 100 the highest general mobility. All data from the ABC Scale and the DEMMI were inserted into a $4 \times 4$ contingency table to compare the objective FR with the subjective self-assessment (FOF) of the participants. The self-assessment was deemed "adequate" if both objective and subjective assessments were either positive (FR and FOF) or negative (no FR and no FOF). Divergences from the concurrent results of the two assessments were deemed "inadequate".

Qualitative data were recorded six months after discharge (T2). Sixteen participants were recruited and interviewed at home regarding their actual mobility, everyday activities and coping strategies due to mobility limitations and FOF. Interviewees were recruited as a convenience subsample in the follow-up and agreed to an interview according to the order in which they were contacted. Four potential participants rejected an interview. The number of subjects was based on the principle of theoretical saturation (17) (18). Theoretical saturation here means the understanding of theoretical saturation according to Glaser and Strauss "Saturation means that no additional data are being found whereby the sociologist can develop properties of the category." (18) All interviews were recorded using audio equipment and transcribed based on predefined transcription rules. The category formation was performed deductively in a first step; based on a literature search was carried out with the keywords fear of falling, dealing with everyday life aspects, walking aids and coping. And based on expert discussions with members of various professions (physiotherapists, doctors, nurses) and affected persons and their relatives. In a second step, the examination units were explored iteratively-inductively. In order to ensure high intercoder reliability, the interviews were coded and categorized by two researchers ("deleted for blinding purposes".) with the help of the coding frame with the categories worked out. Both researchers have extensive experience of planning, conducting and analyzing qualitative interviews. The coding guide was added after reviewing the first four interviews and after reflection by a second rater with anchor examples as well as other categories.. Four interviews with the deductively developed coding guideline were coded, reflected by both researchers and four further categories with definitions, coding rule and anchor example were worked out. Then the already coded four interviews were reencoded, reflected again in the team and the categories were found to be appropriate and a further 12 interviews were coded with the modified coding guideline. Subcategories were worked out and deleted or added in a deductive approach and then elaborated into main categories (coping strategies (active, passive, indifferent) and awareness). The ratio of deductive to inductive approach is nine to four.

The qualitative content analysis was performed according to Mayring. This took the form of a structuring content analysis; more precisely, content structuring in which the material obtained for certain content (here, according to the coding guide including its inductive additions) is extracted and summarized (19).

\section{Results}

Table 1 presents an overview of the sociodemographic variables of the total cohort as well as the subsample used in this publication. Both cohorts demonstrated a larger proportion of women (69\% or 69 in the total cohort, $81.25 \%$ or three in the subsample). Results for age, the DEMMI and the ABC Scale were comparable. 


\begin{tabular}{|c|c|c|c|c|c|}
\hline Quantitative part & & & Qualitative part & & \\
\hline $\mathbf{N}=100$ & & IQR & $\mathrm{N}=16$ & & IQR \\
\hline Sex & & & Sex & & \\
\hline Male (n) & 31 & & Male (n) & 3 & \\
\hline Female (n) & 69 & & Female (n) & 13 & \\
\hline Age (median) & 79.5 years & $65-92$ & Age (median) & 81.5 years & $65-86$ \\
\hline Participants with falls (n) & & & Participants with falls (n) & & \\
\hline & 58 & & & 10 & \\
\hline DEMMI (median) & 62 & $33-100$ & DEMMI (median) & 58.9 & $30-100$ \\
\hline ABC (median) & 29 & $6-47$ & ABC (median) & 26.1 & $6-47$ \\
\hline
\end{tabular}

Table 1: Characteristics of the study sample

Of the 16 seniors interviewed, only six adequately assessed their own FR at T1.

During the 16 interviews at time T2, the main categories of coping strategies, personal coping, coping aid use and awareness of oneselt were worked out. Interviews lasted around 45 minutes on average. Of these, coping strategies and awareness of oneself referred to FOF and are therefore reported in detail (An additional file shows these in more detail [see Additional file 2]).

The category coping strategies could be divided into subcategories that were either active or positively connoted, or passive or negative; additionally, a third subcategory could be identified with indifferent attitudes towards FOF and falls in general. Twelve out of the 16 interviewees reported active/positive coping. Statements such as "That the [rollator walker] is so easily put together. Otherwise, that would make [the connection to the heating pipe] impossible" (007) and "Could have been worse, I think. That I was ending up in a wheelchair or something like that. I should be happy" (042) demonstrated a positive attitude to the challenge of their own FR or the reduced state of health after hospitalization.

Two more interviewed seniors revealed passive/negative coping: "Well, I'm not even thinking about falling down right now" (026) and "But I don't know how it is going to be. You can only pray every day that you will feel better" (079). From these interviews, subcategories such as the lack of prospects or low self-efficacy could be extracted.

In addition, indifferent coping strategies were evident in two seniors who stated neither clearly active/positive nor clearly passive/negative coping: "[...] others do worse, don't they? [...] Well, limited, but I have to move. I need to do my household duties" (049) and "And it's up to me what I make of it" (099).

The main category awareness could be further divided into three subcategories: consciousness about oneself - self-confidence; consciousness about oneself - social network; consciousness about oneself - potential fall cascade.

Merging qualitative and quantitative data, the six participants revealed a correlation between adequate self-assessment of the risk of falling and active/positive coping in the category coping strategies. These interviewees demonstrated an adequate assessment of their own FR and demonstrated an active or positive attitude towards this risk. That was not the case for the people who estimated their own FR inadequately $(\mathrm{n}=10)$. This group showed a variety of characteristics such as "active coping" as well as "passive coping" and "indifferent coping".

\section{FIGURE 2}

In the category awareness, a clear correlation between adequate self-assessment of the risk of falling and the existence of the category consciousness about oneself could be observed. All interviewees who estimated their own FR adequately presented some content during the interview, which allowed for classification into the category awareness of themselves: "So, I'm very unsteady [...] I am very, very slow" (007); "Well, because I actually consciously treat that a little bit; because I don't want to fall" (019). This explicit connection was not observable among those who inadequately assessed their risk of falling (An additional file shows this in more detail [see Additional file 3]). 
The categories personal coping and coping aid use refer to interviewees who reported receiving support from relatives and neighbors and the use of walking aids.

\section{Discussion}

We aimed at identifying different coping strategies in older people regarding their own subjective FR, depending on whether the subjective self-assessment was adequate or inadequate. Within our study subsample, more than a half of the participants interviewed were not able to adequately self-assess their individual risk of falling. These results are much higher than the findings reported in other publications (6-8), where around $22-42 \%$ of the older participants assessed their risk of falling inadequately. Of the 10 participants who inadequately assessed their own FR, six considered the risk of falling at too high a level, whereas four participants perceived their risk of falling to be lower than it objectively was. At this point, we can only speculate why the rate for the inadequate self-assessment of FR was much higher than in prior publications. First, our sample size was much lower and therefore prone to the risk of statistical deviations. For the same reason, the rate of fallers could be higher in our subsample too. Additionally, as falls in the past are known as direct or indirect risks of FOF (20), this could be a possible explanation for the high rate of inadequate self-assessment. However, as our presented results are based on qualitative research, we do not aim at generalizing our findings. Therefore, we do not regard the lack of comparability with other publications using a purely quantitative approach to be problematic.

The demonstrated coping strategies adopted by the interviewees corresponded with the findings of Høst et al. (9), suggesting there are active and passive coping strategies to counter FR. In our study, we additionally encountered an indifferent coping strategy, which is characterized by the relativism and comparison of own risk of falling with a conceivable greater risk of falling. Furthermore, our analysis suggests that an adequate self-assessment of FR is accompanied by positive coping strategies, while an inadequate estimation of the risk of falling is accompanied by several coping strategies.

Another finding of particular interest is that the group of participants, who could adequately assess their own risk of falling, reported on their own awareness of their situation. This has to the best of our knowledge, not been described in prior publications. In our study, this group displayed active/positive as well as awareness coping, which was not the case for the participants who could not properly assess their risk of falling. While these results cannot be generalized due to the qualitative nature of the data, it still offers an interesting insight into potential prerequisites for positively coping with FR, mobility limitations and FOF. As positive coping was associated with more reported everyday activities during the interviews, the ability of an older person to develop a comprehensive awareness regarding his or her situation could constitute a prerequisite for adequately assessing his or her FR, which consequently could pose as a prerequisite for developing or maintaining an active lifestyle based on a positive coping strategy.

Based on this theory, it can be hypothesized that different forms of consultation and support to promote self-awareness will be needed to lower FR for older people who lack an adequate awareness of their own FR. However, as the sample size in this study was very small and based on qualitative data, more research is needed in order to gain a better understanding about the prerequisites needed to develop adequate awareness and how this awareness could be facilitated and measured during any kind of intervention to lower FR in older people.

\section{Conclusion}

Our study suggests that older people who adequately assessed their own FR showed better self-awareness about their situation. These participants-with better self-confidence-were more able to develop positive coping strategies, leading to more activities in their daily lives. More research is needed to more fully understand this phenomenon in order to derive interventional concepts from these findings and to introduce these concepts into existing programs for lowering FR in older people.

\section{Abbreviations And Symbols}

$\mathrm{ABC}$ scale $=$ Activities-specific Balance Confidence Scale

DEMMI = de Morton Mobility Index

FOF $=$ fear of falling

$F R=$ fall risk

$\mathrm{IQR}=$ interquartile range

NYHA IV = New York Heart Association, grade VI

PADD = peripheral arterial occlusive disease

T1 quantitative = time of testing 1, quantitative

T2 qualitative = time of testing 2, qualitative

\section{Declarations}

\section{Ethics approval and consent to participate}


The study was assessed and positively evaluated by the responsible ethics committee (Ethics Number EA4/012/16) on March 16, 2016, prior to commencement. All subjects were informed of the study in written and oral form before inclusion and signed a written consent form before the start of the measurements. The declaration of consent of the participants has been checked by the Ethics Committee of the Charité-Universitätsmedizin Berlin and is available for each participant.

\section{Consent for publication}

Not applicable.

\section{Availability of data and materials}

The datasets used and analyzed during the current study are available from the corresponding author on reasonable request.

\section{Competing interests}

The authors declare that they have no competing interests.

\section{Funding}

Own funding. This work was also supported by the Geriatrics Research Group of Charité Universitätsmedizin Berlin.

\section{Authors' contributions}

NS was the author to develop the study design (mixed methods design) as well as the interview guideline and coding guideline. NS recruited participants, collected data (sociodemographic data, mobility data, data on self-efficacy and fear of falling) and conducted interviews with participants. The data were analyzed for adequate and inadequate self-assessment of the fall risk, the interviews were transcribed, analyzed and interpreted. NS was a major contributor in writing the manuscript.

HB recruited participants, collected data (sociodemographic data, mobility data, data on self-efficacy and fear of falling), analyzed and critically revised data. SM recruited participants, collected data (sociodemographic data, mobility data, data on self-efficacy and fear of falling).

UMW contributed substantially to the development of the study and critically revised the manuscript.

JK created the quantitative part of the study concept and study design and collected mobility data. JK analyzed and interpreted data and developed the manuscript and critically revised the manuscript. JK obtained the ethics vote as study director.

All authors read and approved the final manuscript.

\section{Acknowledgements}

Not applicable.

\section{Authors' information}

The author NS works in Geriatrics Research Group of Charité Universitätsmedizin Berlin.

\section{References}

1. Runge M, Hunter G. Determinants of musculoskeletal frailty and the risk of falls in old age. J Musculoskelet Neuronal Interact. 2006 Jun;6(2):167-73.

2. Varnaccia G, Rommel A. Das Unfallgeschehen bei Erwachsenen in Deutschland [Internet]. Robert Koch-Institut, editor. Beiträge zur Gesundheitsberichterstattung des Bundes; 2013. Available from:

http://www.rki.de/DE/Content/Gesundheitsmonitoring/Gesundheitsberichterstattung/GBEDownloadsB/unfallbericht_geda.pdf\%3F_blob\%3DpublicationF

3. Saß A-C, Wurm S, Ziese T. Beiträge zur Gesundheitsberichterstattung des Bundes. Gesundheit und Krankheit im Alter. Robert Koch Institut; 2009.

4. Rubenstein LZ. Falls in older people: epidemiology, risk factors and strategies for prevention. Age Ageing. 2006 Sep;35 Suppl 2:ii37-41.

5. Filiatrault J, Desrosiers J. Coping Strategies Used by Seniors Going through the Normal Aging Process: Does Fear of Falling Matter? Gerontology. 2011;57:228-236.

6. Delbaere K, Crombetz G, Vanderstraeten G, Willems T, Cambier D. Fear-related avoidance of activities, falls and physical frailty. A prospective communitybased cohort study. Age and Ageing. 2004;(33):368-73. 
7. Kiselev J, Wegel S, Moosburner S, Dimitrovska S, Steinhagen-Thiessen E. Bewertung des Sturzrisikos bei älteren Menschen: Divergenzen zwischen der subjektiven sturzassoziierten Selbstwirksamkeit und objektiven Sturzrisiken. physioscience. 2017 Mar;13(1):25-30.

8. Fortinsky RH, Panzer V, Wakefield D, Into F. Alignment between balance confidence and fall risk in later life: Has over-confidence been overlooked? Health, Risk \& Society. 2009 Aug 1;11(4):341-52.

9. Høst D, Hendriksen C, Borup I. Older people's perception of and coping with falling, and their motivation for fall-prevention programmes. Scandinavian Journal of Public Health. 2011;39:742-8.

10. Lazarus RS. Coping Theory and Research: Past, Present, and Future. Psychosomatic Medicine. 1993;(55):234-47.

11. Powell LE, Myers AM. The Activities-specific Balance Confidence (ABC) Scale. Journal of Gerontology. 1995;50A(1):28-34.

12. Schott N. Deutsche Adaptation der “Activities-Specific Balance Confidence (ABC) Scale„, zur Erfassung der sturzassoziierten Selbstwirksamkeit. Z Gerontol Geriat. 2008 Dec 1;41(6):475-85.

13. Filiatrault J, Gauvin L, Fournier M, Parisien M, Robitaille Y, Laforest S, et al. Evidence of the Psychometric Qualities of a Simplified Version of the Activitiesspecific Balance Confidence Scale for Community-Dwelling Seniors. Archives of Physical Medicine and Rehabilitation. 2007 May 1;88(5):664-72.

14. Lajoie Y, Gallagher SP. Predicting falls within the elderly community: comparison of postural sway, reaction time, the Berg balance scale and the Activitiesspecific Balance Confidence (ABC) scale for comparing fallers and non-fallers. Archives of Gerontology and Geriatrics. 2004 Jan 1;38(1):11-26.

15. Myers AM, Fletcher PC, Myers AH, Sherk W. Discriminative and evaluative properties of the activities-specific balance confidence (ABC) scale. The Journals of Gerontology Series A, Biological Sciences and Medical Sciences. 1998;53(4):287-94.

16. de Morton NA, Davidson M, Keating JL. The de Morton Mobility Index (DEMMI): an essential health index for an ageing world. Health Qual Life Outcomes. 2008 Aug 19;6:63.

17. Glaser BG, Strauss A. Glaser, Barney G. \& Strauss, Anselm (1998). Grounded Theory. Strategien qualitativer Forschung. Bern: Huber. (Orig. 1967: The Discovery of Grounded Theory: Strategies for Qualitative Research. New York: de Gruyter). Bern: Huber; 1998.

18. Glaser BG, Strauss A. The Discovery of Grounded Theory: Strategies for Qualitative Research. New York, NY, US: de Gruyter; 1967.

19. Mayring P. Qualitative Inhaltsanalyse: Grundlagen und Techniken. 11th ed. Weinheim: Beltz; 2010. 144 p.

20. Friedman SM, Munoz B, West SK, Rubin GS, Fried LP. Falls and fear of falling: which comes first? A longitudinal prediction model suggests strategies for primary and secondary prevention. J Am Geriatr Soc. 2002 Aug;50(8):1329-35.

\section{Figures}

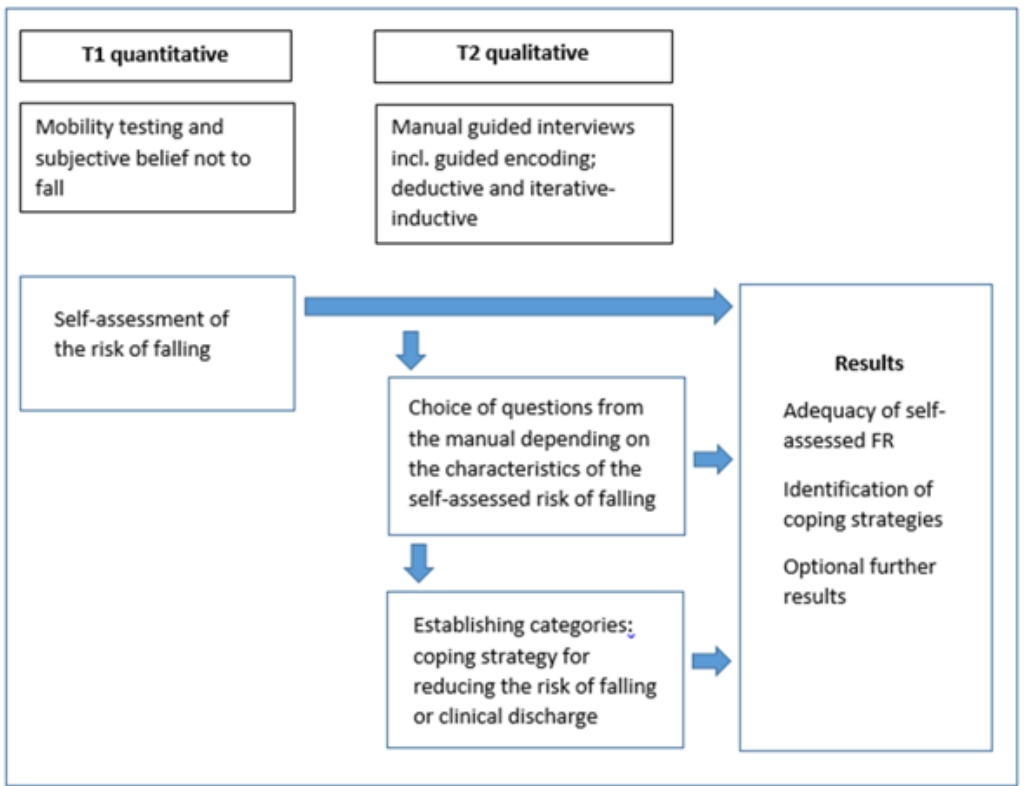

\section{Figure 1}

Sequential design; own representation 


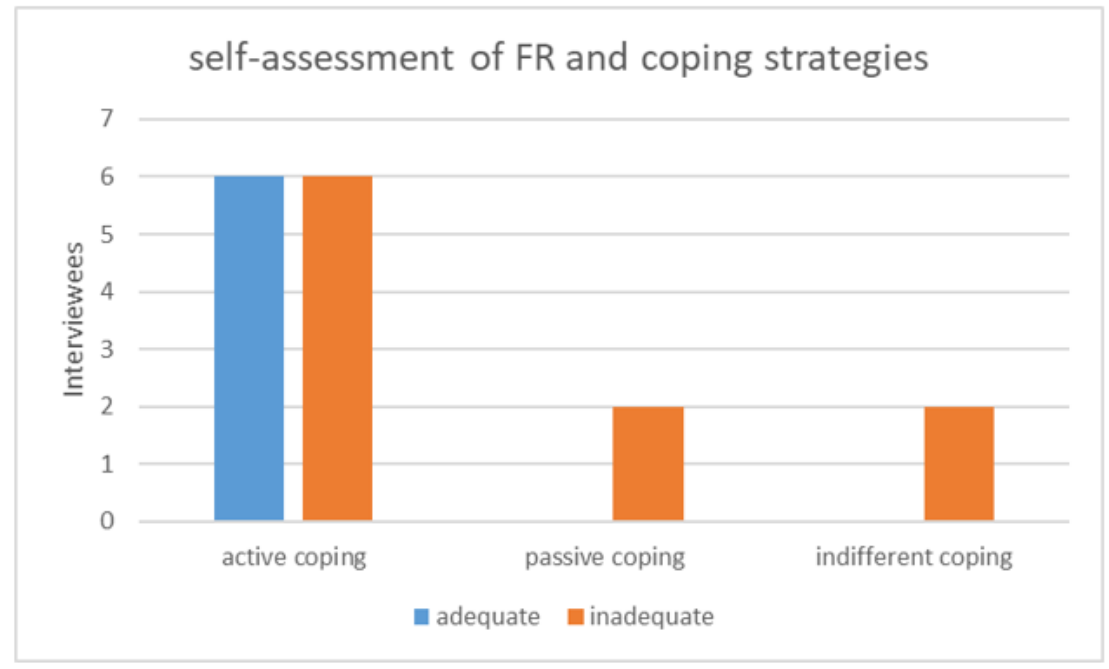

Figure 2

Self-assessment of the FR and characteristics of the category coping strategies; own representation 